prevenção, multifatorial a taxa de lesões no futebol pode ser reduzida. ${ }^{3}$ Heidt e col. selecionaram de forma aleatória 42 jogadoras de futebol de um grupo de 300 jogadoras e aplicaram no grupo experimental um programa de treino antes do início da época durante sete semanas. O programa combinava exercícios específicos de melhoria da condição cardiorrespiratória, treino pliométrico, treino de força e flexibilidade. No final da época desportiva, apenas 14\% das jogadoras se lesionaram, a contrastar com os 34\% do grupo de controlo.

Tendo em consideração que a taxa de lesões nos jogadores de futebol é elevada e que uma fundamentada intervenção multifatorial pode prevenir a ocorrência de lesões, este artigo tem por objetivo apresentar um conjunto de estratégias para prevenção de lesões no jogador de futebol. De facto, o papel do médico de equipa é pivotal no processo preventivo, devendo articular-se com os outros profissionais (especialistas de exercício, fisioterapeutas, treinador) e para isso dominar as metodologias de treino por eles adotadas.

\section{Revisão da Literatura}

A otimização da prevenção de lesões no jogador de futebol exige, para além do domínio da especificidade da modalidade desportiva e das principais lesões associadas, um conhecimento aprofundado das características individuais de cada jogador (historial clínico, dados pós avaliação musculoesquelética e funcional) e dos princípios de treino para uma adequada periodização de treino. De seguida, apresentar-se-ão algumas das estratégias a adotar para se conseguir que se previnam lesões desportivas e, necessariamente para a otimizar o rendimento desportivo de um futebolista. A saber:

\section{- Avaliação Funcional}

Para eficazmente podermos prescrever exercícios corretivos ou de otimização da performance desportiva, complementares ao treino técnico-tático de futebol, teremos de avaliar o atleta. Uma das avaliações que se deverá realizar é a avaliação postural dinâmica. Um possível teste de se realizar é o Overhead Squat 
(Figura 1), em que a análise da qualidade do movimento na vista anterior, lateral e posterior permite recolher dados importantes, tais como: varo ou valgo dos joelhos, manutenção ou não do tronco em posição neutra, elevação ou não do calcanhar, etc. ${ }^{5}$

Outra bateria de testes que se pode utilizar é o Functional Movement Screening (FMS) (Figura 2). Este conjunto de testes pretende avaliar a qualidade dos padrões de movimento fundamentais para que se possa identificar presumíveis limitações ou assimetrias do atleta. O FMS inclui sete testes que são cotados em uma escala ordinal de 0 a 3. Os sete testes são: agachamento, transpor um obstáculo, afundo, mobilidade do ombro, flexão coxofemoral de modo ativo em decúbito dorsal, push-up e estabilidade na rotação. ${ }^{6}$

A pontuação de 3 indica que o movimento foi concluído conforme as instruções e não apresenta qualquer compensação de movimento e dor. O score de 2 indica que o atleta completou o movimento livre de dor mas com algum nível de compensação, e a pontuação de 1 indica que o atleta não conseguiu completar o movimento conforme as instruções. Um 0 é atribuído se o atleta experimenta dor em qualquer parte do movimento. Dos sete testes que compõem o FMS, cinco deles (transpor um obstáculo, afundo, mobilidade do ombro, flexão coxofemoral de modo ativo em decúbito dorsal e estabilidade na rotação) são realizados e classificados separadamente para os lados direito e esquerdo do corpo. Ao atribuir a pontuação para um teste, que incorpora o lado esquerdo e direito, o menor dos dois resultados é utilizado para a pontuação final. A pontuação geral FMS pode variar de 0 a $21 .^{7}$

Vários estudos têm utilizado o valor de 14 como limite crítico, enquanto preditor de lesão $0^{8,9} \mathrm{e}$ referem que o risco de lesão é superior em atletas cuja pontuação total é inferior a 14. ${ }^{7,8}$ Kiesel e col. analisaram, retrospectivamente, a relação entre a pontuação FMS para jogadores de futebol americano e a probabilidade de lesão. As pontuações FMS foram obtidas na pré-temporada em 46 jogadores. Concluiu-se que a probabilidade de lesão foi 11,7 vezes superior em jogadores com uma pontuação total inferior a 14 pontos. $^{8}$

O objetivo da avaliação funcional com bateria de testes da FMS, o overhead squat ou vários outros testes, tais como o teste de equilíbrio unipodal, destina-se a determinar a existência de alguma falha na estratégia de estabilização e/ ou movimento do jogador para atuação preventiva.

\section{- Respeitar os rácios de força fun- cional ideais do corpo}

No trem inferior deverá existir uma relação ótima de 100-60/70\% (valores de força concêntrica) entre musculatura posterior e anterior da coxa. Ou seja, os músculos quadricípites deverão ser capazes de produzir valores mais elevados de força dos que os músculos isquioperoniotibiais (IPT), mas estes últimos não deverão ser bastante mais fracos que os primeiros, o que sucede muitas das vezes. A alteração desta relação coloca os músculos posteriores da coxa e o ligamento cruzado anterior em maior risco de lesão. A relação ótima é de 1:1 na comparação entre o valor de força excêntrica dos músculos IPT e o valor de força concêntrica do quadricípite. ${ }^{10}$ Na perspetiva de contrariar a fragilidade dos músculos posteriores da coxa dos jogadores de futebol, sugere-se a inclusão de sessões de treino de força para esta musculatura numa fase preparatória da época desportiva e também posteriormente, respeitando os princípios de progres- sividade e adaptação durante o período competitivo, com intensidade e volume ajustados. Alguns dos exercícios que poderão ser realizados são o exercício nórdico ou o exercício de leg curl. Os jogadores com valores acima dos limites críticos de força na relação isquioperoniotibiais / quadricípite têm menor número de ocorrências e de reincidências de lesões musculares dos músculos isquiotibiais. ${ }^{11}$ Numa perspetiva mais holística de reforço de musculatura do trem inferior, o agachamento pode ser considerado uma ótima opção de treino de força, devendo os atletas, sempre que a situação clínica o permita, realizar uma grande amplitude de movimento (agachamento completo) para maior participação muscular do grande glúteo. ${ }^{12}$

\section{- Treino de equilíbrio}

Os exercícios de equilíbrio, realizados com ou sem superfícies instáveis, previnem e auxiliam na recuperação de lesões a nível da

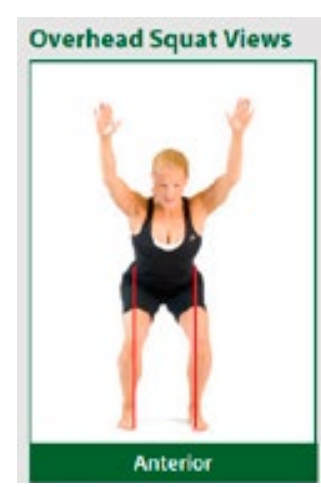

Figura 1 - O Overhead Squat

Ref. https://dtaOyqvfnusiq.cloudfront.net/thefitnesstraineracademy/2015/10/image04.png
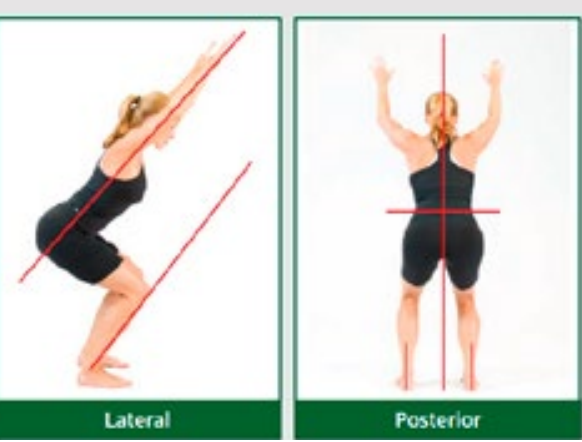

\section{The Functional Movement Screen}

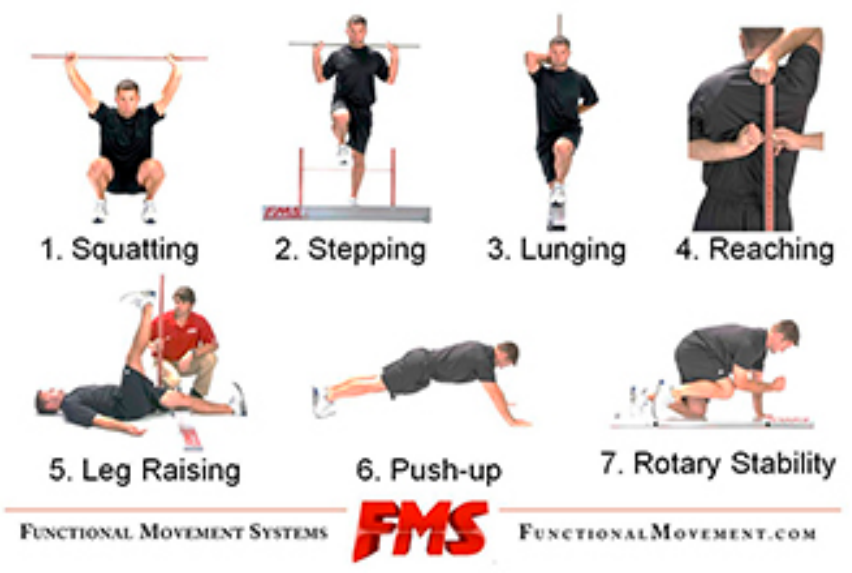

Figura 2 - Os testes do Functional Movement Screening Ref. https://www.physio-pedia.com/images/6/60/FMS.jpeg 
tibiotársica. No estudo de Mchugh foi avaliada a incidência da entorse no tornozelo por trauma indireto, antes e depois da intervenção, em 175 jogadores de futebol. ${ }^{13} \mathrm{~A}$ intervenção teve a duração de três anos e consistiu num protocolo de treino de equilíbrio e de estabilidade num rolo de espuma. Em cada época desportiva este protocolo foi aplicado por 5 dias por semana, durante 4 semanas na pré-temporada e duas vezes por semana durante a temporada. Os resultados obtidos demonstraram que existiu redução das ocorrências de entorse de tornozelo após a intervenção, o que está de acordo com resultados obtidos por outros

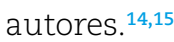

O treino de equilíbrio permite a diversificação dos estímulos sensoriais aplicados ao atleta, a melhoria da propriocetividade e do controlo postural. ${ }^{15}$ Consegue-se, concomitantemente, uma transferência positiva para as situações reais de treino e de competição, nas quais existe por vezes alguma irregularidade do terreno de jogo e abordagem e contacto do pé com solo diversa. Resumindo, a inclusão de treino de equilíbrio e de exercícios que desafiam o controlo neuromuscular vai permitir a prevenção de lesões no joelho e tornozelo.

\section{- Treino do Core}

TSE M e at al referem que o Core engloba o conjunto de músculos da pélvis e do tronco (Figura 3), responsáveis por manter a estabilidade da coluna vertebral e da bacia, os quais são críticos para a transferência de

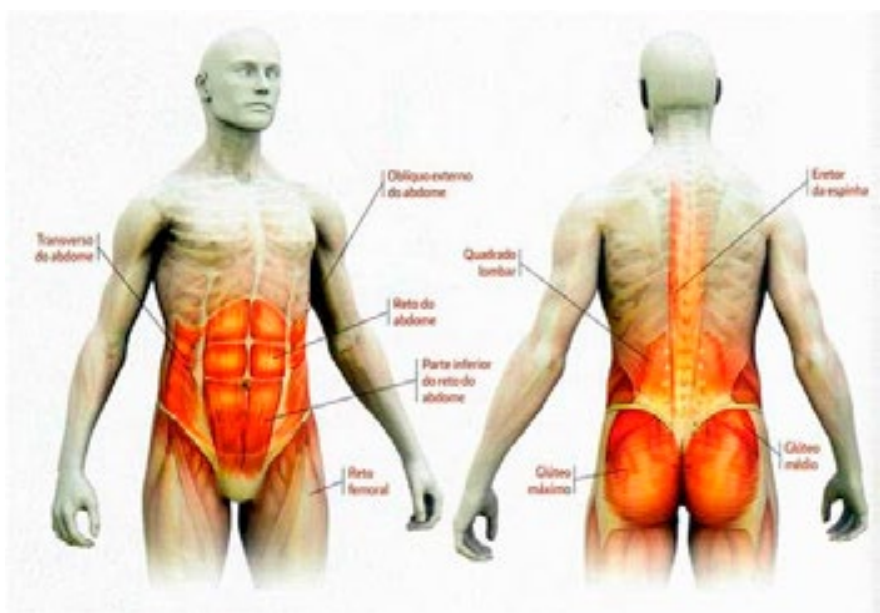

Figura 3 - Músculos do core

Ref. https://dicasdemusculacao.org/wp-content/uploads/musculos-do-core.jpg energia desde o tronco para as extremidades. ${ }^{16}$ Destaca-se um conjunto de músculos predominantemente constituídos por fibras lentas, mais profundos e que têm ação local, tais como o transverso do abdómen, o multifidus, o pequeno oblíquo, o transverso do abdómen, os músculos diafragmáticos e os músculos do pavimento pélvico, e uma musculatura mais superficial com maior proporção de fibras rápidas. Neste último grupo identificamos o grande oblíquo, o reto abdominal, o quadrado lombar e os eretores da coluna. Incluem-se ainda, enquanto constituintes do core, os músculos da região da anca, nomeadamente o grande e o médio glúteo. Os músculos identificados, sejam do tronco ou da região da anca, terão de ser fortes e funcionais para darem estabilidade ao centro. A preservação desta estabilidade da região central do tronco será depois essencial para o bom funcionamento das extremidades inferiores.

No futebol, o típico exercício muitas vezes prescrito de flexão sucessiva do tronco, vulgo abdominal para treinar reto abdominal não serve as necessidades dos jogadores e pode aumentar risco de lesão na coluna lombar. ${ }^{17}$ Já existe atualmente um conjunto de exercícios diferenciados que permite um treino eficaz da musculatura do core, como por exemplo o pallof press, o agachamento, o levantamento terra, o lift, entre outros.

\section{- Treino de pliometria}

Na maioria dos gestos desportivos, e no futebol não é exceção, os músculos não funcionam de forma puramente concêntrica, excêntrica ou isométrica, mas funcionam num ciclo muscular de alongamento-encurtamento. Ao alongamento muscular sucede-se a fase de encurtamento (exemplo da corrida ou salto). Ou seja, o ciclo muscular de alongamento-encurtamento caracteriza-se pela produção de uma ação dinâmica concêntrica potente que é imediatamente antecedida de uma ação dinâmica excêntrica do mesmo músculo e é treinável através de exercícios pliométricos. O objetivo do treino de pliometria é diminuir o tempo entre o final da contração muscular excêntrica e o início da contração concêntrica. Este tipo de treino visa conferir a capacidade de treinar padrões de movimento específicos biomecanicamente corretos, fortalecendo o músculo, o tendão e o ligamento de forma mais funcional. Deste modo, associada ao treino de agilidade, a pliometria terá uma ação importante na prevenção de lesões ligamentares no joelho e tornozelo. ${ }^{18}$ Por outro lado, a pliometria permite melhorar os valores obtidos no salto vertical, velocidade e potência muscular nos jogadores de futebol. ${ }^{19}$ Certamente que este aumento dos valores de força permitirá melhorar a economia de esforço e minorar a probabilidade de lesão.

\section{- Utilização da libertação miofascial antes do treino ou competição}

Para a libertação miofascial auto-induzida tem-se utilizado os rolos terapêuticos, ou foam rollers, que são de utilização simples e dispensam ajuda externa. Este método de treino de flexibilidade diminui as adesões fasciais, previne e corrige encurtamentos musculares, contribui para a correção de alterações posturais e permite a melhoria da amplitude de movimento e da força. ${ }^{20,21}$ Fundamenta-se que a pressão aplicada pelo rolo terapêutico vai desativar o nódulo através da estimulação dos órgãos tendinosos de Golgi, num processo que se denomina de inibição autogénica. Deste modo, consegue-se restaurar o sistema miofascial, eliminando os mecanismos propriocetivos desencadeadores da dor/disfunção. ${ }^{5}$

Os jogadores de futebol poderão aplicar as técnicas de libertação miofascial nos grupos musculares em que existam os nódulos ou adesões musculares, na fase de aquecimento, antes do trabalho cardiorrespiratório ou na fase de retorno à 
calma. Neste momento, o objetivo será o de minimizar a dor muscular pós-esforço ${ }^{22}$, aconselhando-se a utilização com baixa intensidade.

\section{- Importância do aquecimento}

O aquecimento deverá preceder a parte fundamental e, entre outros benefícios, permitirá aumentar a temperatura corporal, a extensibilidade do tecido conjuntivo e o fluxo sanguíneo ao músculo cardíaco e aos músculos solicitados. Para além da possível inclusão da libertação miofascial, o aquecimento deverá incluir uma parte geral que incluirá exercícios gerais, multiplanares, tais como corrida, corrida lateral, por exemplo, e uma parte específica já com exercícios orientados para a tarefa principal. ${ }^{18}$ Aqui poder-se-ão incluir exercícios de equilíbrio, de pliometria e de ativação muscular.

\section{Conclusões}

A identificação dos fatores de risco e das lesões mais prevalentes nos jogadores de futebol permitirá ao clínico uma abordagem mais direcionada para a prevenção de lesões. Esta deverá ser holística e contar com a colaboração de vários outros profissionais, como o especialista de exercício, preparador físico, fisioterapeuta e treinador, tendo em consideração aspetos tão cruciais quanto a realização inicial de uma avaliação funcional, a qual permitirá a posterior correção de assimetrias e de padrões de movimento, a inclusão suplementar de treino do Core, de equilíbrio e pliométrico e a utilização de técnicas de libertação miofascial. Ao clínico competirá conhecer estes conceitos e coordenar o processo preventivo. Aliado ao conhecimento dos princípios do treino, a adopção do conjunto de estratégias apresentadas permitirá reduzir o número e a gravidade das lesões sofridas por jogadores de futebol.

Os autores negam qualquer conflito de interesses

Correspondência

Rodrigo Miguel Arsénio dos Santos Ruivo rodrigoruivo@clinicadasconchas.pt

\section{Bibliografia}

1. Brito J, Malina RM, Seabra A, Massada JL, Soares JM, Krustrup P, et al. Injuries in Portuguese youth soccer players during training and match play. J Athl Train [Internet]. 2012; 47(2):191-7. Available from: http://www. pubmedcentral.nih.gov/articlerender.fcgi? artid=3418131\&tool=pmcentrez\&renderty pe=abstract

2. Ekstrand J, Hägglund M, Waldén M. Injury incidence and injury patterns in professional football: the UEFA injury study. Br J Sports Med [Internet]. 2011 Jun [cited 2014 Jan 22];45(7):553-8. Available from: http://www. ncbi.nlm.nih.gov/pubmed/19553225

3. Ekstrand J, Gillquist J LS. Prevention of soccer injuries: supervision by doctor and physiotherapist. Am J Sport Med. 1983; (11):116-20.

4. Heidt R, Sweeterman L, Carlonas R. Avoidance of soccer injuries with preseason conditioning. AmJ Sport. 2000; 28:659-62.

5. Clark M, Lucett S. NASM Essentials of corrective exercise training. Baltimore: LIppincott Williams\&Wilkins; 2011.

6. Kiesel K, Hall WG. Can serious injury in professional football be predicted by a preseason fucntionak movement screen? North Am J Sport Phys Ther. 2007; 2(3):147-58.

7. Connor FGO, Deuster PA, Davis J, Pappas CG, Knapik JJ. Functional Movement Screening Predicting. 2011; 2224-30.

8. Kiesel K, Plisky P, Voight M. Can serious injury in professional football be predicted by a preseason functional movement screen? Am J Sport Phys Ther. 2007; 2(3):147-50.

9. Kiesel K, Plisky P, Butler H. Functional movement test scores improve following a standardized off-season intervention program in professional football players. Scand J Med Sci Sport. 2011; 21(2):287-92.

10. Ruivo R. Manual de Avaliação e Prescrição de Exercício. 3. ${ }^{\circ}$ Ed. Self, editor. Lisboa; 2015.

11. Croisier J. Factors associated with recurrent hamstring injuries. Sport Med. 2004; 34:662-95.

12. Caterisano A. The effect of back squat depht on the Emg activity of 4 superficial hip and thigh muscles. J Strenght Cond Res. 2002; 16(3):428-32

13. Mchugh MP, Tyler TF, Mirabella MR, Mullaney MJ, Nicholas SJ. The Effectiveness of a Balance Training Intervention in Reducing the Incidence of Noncontact Ankle Sprains in High School Football Players. Am J Sports Med. 2007; 35(8):1289-94.

14. Wedderkopp N, Kaltoft M, Lundgaard B, Rosendahl M, Froberg K. Prevention of injuries in young female players in European team handball: a prospective intervention study. Scand J Med Sci Sport. 1999; 9:41-7.

15. Verhagen E, Beek A, Twisk J, Bouter L, Bahr $\mathrm{R}$. The Effect of a Proprioceptive Balance Board Training Program for the prevention of ankle sprains - a prospective controlled trial. Am J Sports Med. 2004; 32(6):1385-93.

16. Tse M, McManus R. Development and validation of a core endurance intervention program: implications for performance in college-age rowers. J Strength Res. 2005; 19:547-52.

17. McGill S. Core Training: Evidence Translating to Better Performance and Injury Prevention. Strength Cond J. 2010; 32:33-46.
18. Mario B, Astrid J, Jiri D. FIFA's Medical Assessment and Research Centre. The "11+" Manual A complete warm-up programme to prevent injuries. Zurique: Vogt-Schild/Druck; 2005;

19. Chelly M, Ghenem M, Abid K, Hermassi S, Tabka Z, Shephard R. Effects of in-season short-term plyometric training program on leg power, jump and sprint performance of soccer players. J Strength Cond Res. 2010; 24(10):2670-6.

20. Haas C, Butterfield T, Zhao Y. Dose-dependency of massage-like compressive loading on recovery of active muscle properties following eccentric exercise: rabbit study with clinical relevance. Br J Sport Med. 2013; 83-8.

21. Schroeder AN, Best TM. Is Self Myofascial Release an Effective Preexercise and Recovery Strategy? A Literature Review. 2015;14(3).

22. Healey K, Hatfield D, Blandpied P. The effects of myofascial release with foam rolling on performance. J Strength Cond Res. 2013; 28:61-8. 